\title{
Relationship between quality of life and anxiety aspects in chronic spontaneous urticaria
}

\author{
Anita Oles-Krykowska¹, Karina Badura-Brzoza², Zenon K. Brzoza ${ }^{3,4}$
}

${ }^{1}$ Clinic of Allergology, Rybnik, Poland

2Department of Psychiatry, School of Medicine with the Division of Dentistry in Zabrze, Medical University of Silesia in Katowice, Poland

${ }^{3}$ Department of Internal Diseases and Allergology, Institute of Medicine, University of Opole, Opole, Poland ${ }^{4}$ Department of Internal Diseases, Allergology and Clinical Immunology, School of Medicine with the Division of Dentistry in Zabrze, Medical University of Silesia in Katowice, Poland

Adv Dermatol Allergo

DOI: https://doi.org/10.5114/ada.2019.85811

\begin{abstract}
Introduction: Clinical experience indicates the role of the psychological component in chronic spontaneous urticaria (CSU). The CSU can lead to psychological disturbances. This phenomenon results in a possible quality of life impairment among CSU patients.

Aim: To analyse in detail the quality of life parameters and anxiety aspects in relation to CSU duration and symptom intensity.

Material and methods: The patient sample was composed of 67 CSU subjects. For the quality of life and mental status assessment, all subjects were questionnaired with $\mathrm{CU}-\mathrm{Q}_{2} \mathrm{OL}$ and State and Trait Anxiety Inventory (STAI). In the control group, 42 healthy volunteers were enrolled to fill in the STAI questionnaire. The Urticaria Activity Score (UAS) was used to assess disease activity.

Results: We observed greater intensity of anxiety as a state and as a trait in CSU patients and greater intensity of anxiety as a state due to increasing patients' age. The CU- $\mathrm{Q}_{2}$ oL Itching subscale was significantly impaired as a result of the intensity of the UAS scale. The Swelling/Mental status subscale was related to anxiety as a state and as a trait. Additionally, we noticed a greater quality of life impairment in subjects with longer disease duration. Conclusions: Our results prove that CSU results in a significant quality of life impairment. This phenomenon particularly concerns patients with longer disease duration time. As anxiety is a relatively frequent disturbance and negatively influences quality of life, we should identify these patients and monitor this parameter especially in older patients.
\end{abstract}

Key words: chronic urticaria, quality of life, anxiety, CU-Q2oL, STAI.

\section{Introduction}

Chronic spontaneous urticaria (CSU) is characterized by the spontaneous occurrence of itchy wheals and/or angioedema for at least 6 weeks. The intensity of urticaria symptoms is subject to monthly, weekly and daily fluctuations. Clinical experience shows the role of the psychological component in urticaria pathogenesis. As urticaria patients are affected by recurrent wheals or oedema often on the uncovered skin, this disorder has an impact on the appearance and severely debilitates daily activity. Additionally, it adversely influences patients' self-perception and provokes some emotional and social problems. Pruritus in the course of CSU often disturbs the sleep cycle. Previous analyses demonstrated that urticaria patients are affected by psychiatric comorbidity and psychological distress [1, 2]. Reoccurrence of urticarial wheals causes emotional disturbances and reduction in social activity [3, 4]. Chronic spontaneous urticaria can lead to psychological disturbances such as anxiety [5]. This phenomenon presumably results in a significant reduction in the quality of life among CSU patients [1]. The adverse influence of CSU on the quality of life was previously confirmed [1, 6]. Impairment of the quality of life in chronic urticaria is comparable to impairment due to coronary artery disease and is higher compared to other dermatologic disorders such as psoriasis, acne and vitiligo $[3,7]$. Quality of life instruments assess subjective perception of the disease impact. Bearing in

Address for correspondence: Zenon K. Brzoza MD, PhD, Department of Internal Diseases and Allergology, Institute of Medicine, University of Opole, al. Witosa 26, 45-401 Opole, Poland, phone: +48 7745206 04, e-mail: zbrzoza@mp.pl Received: 26.03.2019, accepted: 9.05.2019. 
mind the prevalence of CSU, it was necessary to prepare a new tool specifically designed for urticaria and its national translations. The only tool prepared specifically for urticaria is CU- $\mathrm{Q}_{2}$ oL that was developed in Italy [8]. Afterwards Spanish, German, Polish, Brazilian-Portuguese, Turkish, Persian and Israeli versions of this questionnaire were elaborated [9-15]. Patient-reported outcomes in CSU can be assessed with the Urticaria Activity Score (UAS) validated for monitoring disease activity in CSU patients [16].

\section{Aim}

The aim of our study is to analyse quality of life parameters and anxiety aspects in more detail in relation to CSU duration and symptom intensity.

\section{Material and methods}

The patient sample was composed of 67 subjects (43 females and 24 males, median age: 45 years; range: 20-58) with active CSU. The diagnosis of CSU was established on the basis of the precise medical history and complex physical examination. The exclusion criteria were other somatic or psychiatric diseases. All patients were treated according to the current guidelines [17]. For the quality of life and mental status assessment, all subjects were requested to complete the Polish version of the $C U-\mathrm{Q}_{2}$ oL questionnaire and the validated and reliable State and Trait Anxiety Inventory (STAI). The Polish version of $\mathrm{CU}-\mathrm{Q}_{2} \mathrm{OL}$ is composed of 23 questions grouped in six subscales: Itching, Swelling/Mental status, Functioning, Sleep, Eating/Limits and Embarrassment. The STAI questionnaire includes 20 questions related to the state of anxiety (X1) and 20 questions assessing anxiety as a trait (X2). Additionally, the questionnaire package included the information letter and the consent form. Informed consent was obtained from all individual participants included in the study. Furthermore, 42 healthy

Table 1. Values of CU- $\mathrm{Q}_{2}$ oL subscales, total quality of life score (Total), Urticaria Activity Score seven day assessment (UAS7), disease duration time (Time) in chronic spontaneous urticaria patients

\begin{tabular}{lccc}
\hline Variable & Median & Min. & Max. \\
\hline Itching & 75.0 & 12.5 & 100 \\
\hline Swelling/Mental status & 50.0 & 5.0 & 80.0 \\
\hline Functioning & 41.67 & 0.0 & 91.67 \\
\hline Sleep & 40.62 & 0.0 & 100 \\
\hline Eating/Limits & 28.12 & 0.0 & 93.75 \\
\hline Embarrassment & 75.0 & 0.0 & 100 \\
\hline Total & 38.0 & 18.0 & 67.0 \\
\hline UAS7 & 18.0 & 8.0 & 31.0 \\
\hline Time [months] & 18.0 & 3.0 & 180 \\
\hline
\end{tabular}

volunteers in the control group (25 females and 17 males, median age: 47 years; range: 19-56) were enrolled to fill in the STAI questionnaire. UAS was used to assess disease activity. This questionnaire analyses the number of wheals and the intensity of pruritus. A recommended 7-day monitoring period (UAS7) results in a summary score of 0-42. Additionally we calculated disease duration time.

\section{Ethics}

The study protocol was approved by the Bioethics Committee of the Medical University of Silesia in Katowice, Poland.

\section{Statistical analysis}

In statistical analyses, the U Mann-Whitney, logistic regression and Spearman tests were used. $P$-values $<0.05$ were considered statistically significant (Statsoft Inc., Statistica 12.0).

\section{Results}

Analysed parameters values are presented in Table 1. We observed greater intensity of anxiety as a state and as a trait in CSU patients as compared to the control group (Figure 1). We demonstrated greater intensity of anxiety as a state due to increasing age of patients (regression coefficient $R=0.34 ; p=0.01$ ) and significant impairment of the Itching subscale as a result of the intensity of the UAS7 scale (regression coefficient $R=0.39 ; p=0.02$ ). The Swelling/Mental status subscale was related to anxiety

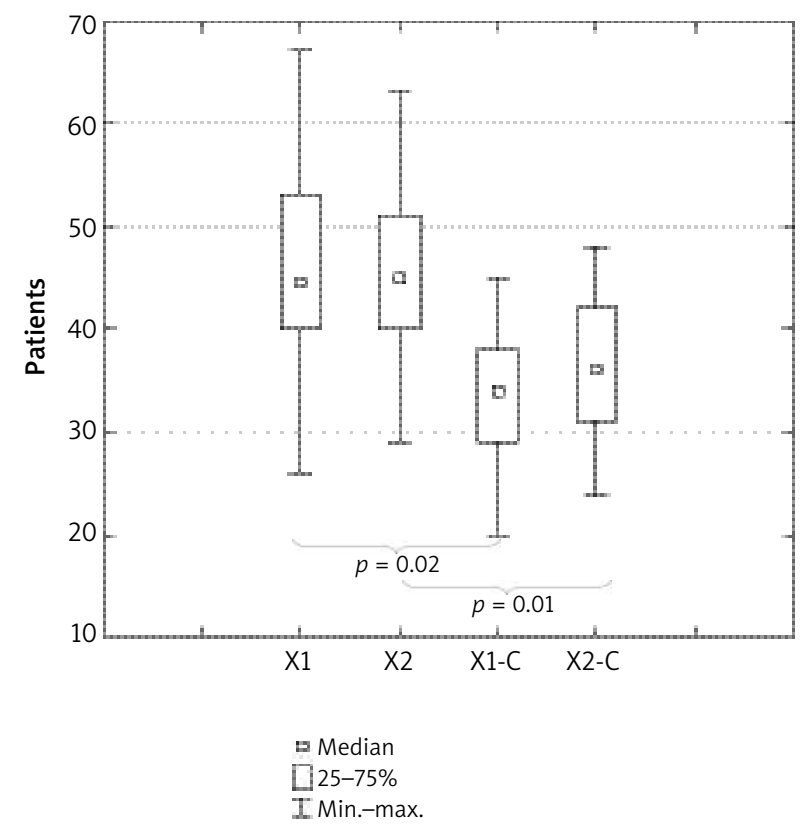

$\mathrm{X} 1$ - anxiety as a state, $\mathrm{X} 2$ - anxiety as a trait, C - control group.

Figure 1. Anxiety levels in examined groups 
as a state $(R$ Spearman $=0.39 ; p=0.01)$ and as a trait ( $R$ Spearman $=0.40 ; p=0.007$ ). Additionally, we noticed a greater total quality of life impairment in subjects with longer disease duration ( $R$ Spearman $=0.30 ; p=0.03$ ).

\section{Discussion}

A high prevalence of psychiatric disturbances in patients affected by skin diseases is relatively well documented [18]. The role of the emotional component in urticaria is emphasised in clinical practice. Chronic spontaneous urticaria is a relatively common dermatosis in allergological and dermatological practice. As other chronic diseases, CSU can be a source of potential psychological problems. Contrary to a significant number of studies on the influence of other skin diseases on the quality of life and psychiatric comorbidity, the number of studies associated with the above problems observed in urticaria is lower. Urticaria patients frequently present with psychiatric comorbidity. Additionally, the adverse influence on the quality of life of such patients was demonstrated [1]. The results of the previous studies indicate that patients with CSU frequently complained of fatigue, lack of sleep or insomnia, which is often due to itching that accompanies wheals [5]. Patients with CSU have been reported to present with high rates of mental disorders [19]. Anxiety and depression have been found to be the most prevalent mental disorders among CSU patients [20]. According to Staubach et al. [1], one in two CSU patients is affected by anxiety, depression and somatoform disorders. As anxiety is a complex disturbance presented as a state or as a trait (i.e. individual tendency to appraise some situations as threatening) we wanted to investigate precisely which aspects of anxiety were manifested in CSU and what group of patients was particularly susceptible. In our study we proved that CSU symptoms result in the occurrence of anxiety, especially in older patients. It must be underlined that it is related to anxiety as a state but not as a trait. We can conclude that anxiety in the course of urticaria is an effect of the disease and not a personal predisposition.

Psychiatric comorbidities were found to be a relevant cofactor for the quality of life impairment in CSU patients. Levels of depression and anxiety are correlated with a decreased quality of life in CSU patients [1]. Barbosa et al. [21] reported a negative influence of anxiety on the general quality of life with a particular impact on some aspects which, however, were assessed with a more general tool than $\mathrm{CU}-\mathrm{Q}_{2} \mathrm{OL}$. In our study we proved that the Swelling/Mental status domain was particularly related to anxiety in both analysed aspects of this disturbance. Longer disease duration particularly favours impairment in the quality of life. In previous studies UAS seemed to be significantly related to quality of life impairment when measured using general dermatological tools such as Dermatology Life Quality Index in CSU patients [8]. Our study demonstrates that the intensity of urticaria symptoms as assessed with the UAS scale reflects in particular the impairment in the Itching subscale. We found a high prevalence of anxiety symptoms in CSU patients. Our results are similar to other research, however, provide more precise information on various aspects of anxiety and the impairment in the quality of life domains in CSU. Our study is novel as it uses a relatively new disease-specific tool that allows for more precise and detailed analyses and brings a new light on particular areas of disease-related adverse effects. Patients with CSU should be investigated for mental disturbances which markedly influence the quality of life. This should be considered in daily practice and in clinical trials.

\section{Conclusions}

As anxiety is a relatively frequent disturbance, we should identify these patients and monitor this parameter, especially in older patients. Further studies are warranted on detailed aspects of quality of life impairment in chronic urticaria. These analyses are possible when a disease-specific tool is used.

\section{Conflict of interest}

The authors declare no conflict of interest.

\section{References}

1. Staubach P, Eckhardt-Henn A, Dechene M, et al. Quality of life in patients with chronic urticaria is differentially impaired and determined by psychiatric comorbidity. Br J Dermatol 2006; 154: 294-8.

2. Brzoza Z, Kasperska-Zajac A, Badura-Brzoza K, et al. Decline in dehydroepiandrosterone sulfate observed in chronic urticaria is associated with psychological distress. Psychosom Med 2008; 70: 723-8.

3. O'Donnell BF. Urticaria: impact on quality of life and economic cost. Immunol Allergy Clin N Am 2014; 34: 89-104.

4. Kang MJ, Kim HS, Kim HO, Park YM. The impact of chronic idiopathic urticaria on quality of life in Korean patients. Ann Dermatol 2009; 21: 226-9.

5. Weldon DR. Quality of life in patients with urticaria. Allergy Asthma Proc 2006; 27: 96-9.

6. Baiardini I, Giardini A, Pasquali M, et al. Quality of life and patients' satisfaction in chronic urticaria and respiratory allergy. Allergy 2003; 58: 621-3.

7. Poon E, Seed PT, Greaves MW, Kobza-Black A. The extent and nature of disability in different urticarial conditions. $\mathrm{Br}$ J Dermatol 1999; 140: 667-71.

8. Baiardini I, Pasquali M, Braido F, et al. A new tool to evaluate the impact of chronic urticaria on quality of life: chronic urticaria quality of life questionnaire (CU-Q2oL). Allergy 2005; 60: 1073-8.

9. Valero A, Herdman M, Bartra J, et al. Adaptation and validation of the Spanish version of the Chronic Urticaria Quality of Life Questionnaire (CU-Q2oL). J Investig Allergol Clin Immunol. 2008; 18: 426-32.

10. Młynek A, Magerl M, Hanna M, et al. The German version of the Chronic Urticaria Quality-of-Life Questionnaire: fac- 
tor analysis, validation, and initial clinical findings. Allergy 2009; 64: 927-36.

11. Brzoza Z, Badura-Brzoza K, Mtynek A, et al. Adaptation and initial results of the Polish version of the GA[2]LEN chronic urticaria quality of life questionnaire (CU-Q(2)oL). J Dermatol Sci 2011; 62: 36-41.

12. Dias GA, Pires GV, Valle SO, et al. Cross-cultural adaptation of the Brazilian-Portuguese version of the chronic urticaria quality-of-life questionnaire - CU-Q2oL. Allergy 2011; 66: 1487-93.

13. Kocatürk E, Weller K, Martus P, et al. Turkish version of the chronic urticaria quality of life questionnaire: cultural adaptation, assessment of reliability and validity. Acta Derm Venereol 2012; 92: 419-25.

14. Tavakol M, Mohammadinejad P, Baiardini I, et al. The Persian version of the chronic urticaria quality of life questionnaire: factor analysis, validation, and initial clinical findings. Iran J Allergy Asthma Immunol 2014; 13: 278-85.

15. Kessel A, Graif Y, Vadasz Z, et al. Adaptation and validation of the Israeli version of the Chronic Urticaria Quality of Life Questionnaire (CU-Q2oL). Isr Med Assoc J 2016; 18: 461-5.

16. Młynek A, Zalewska-Janowska A, Martus P, et al. How to assess disease activity in patients with chronic urticaria? Allergy 2008; 63: 777-80.

17. Zuberbier T, Aberer W, Asero R, et al. The EAACI/GA(2) LEN/ EDF/WAO Guideline for the definition, classification, diagnosis, and management of urticaria: the 2013 revision and update. Allergy 2014; 69: 868-87.

18. Evers AW, Lu Y, Duller P, et al. Common burden of chronic skin diseases? Contributors to psychological distress in adults with psoriasis and atopic dermatitis. Br J Dermatol 2005; 152: 1275-81.

19. Uguz F, Engin B, Yilmaz E. Axis I and Aixis II diagnoses in patients with chronic idiopathic Urticaria. J Psychosom Res 2008; 64: 225-9.

20.Pasaoglu G, Bavbek S, Tugcu H, et al. Psychological status of patients with chronic urticaria. J Dermatol 2006; 33: 765-71.

21. Barbosa F, Freitas J, Barbosa A. Chronic idiopathic urticaria and anxiety symptoms. J Health Psychol 2011; 16: 1038-47. 